\title{
Core-Collapse Supernovae in the Early Universe: Radiation Hydrodynamics Simulations of Multicolor Light Curves
}

\begin{abstract}
The properties of the first generation of stars and their supernova (SN) explosions remain unknown due to the lack of their actual observations. Pop III stars may have been very massive and predicted to be exploded as pair-instability SNe, but the observed metal-poor stars show the abundance patterns which are more consistent with yields of core-collapse SNe. We study the multicolor light curves for a metal-free core-collapse SN models (massive stars of 25100 solar mass range) to determine the indicators for the detection and identification of first generation SNe. We use mixing-fallback supernova explosion models which explain the observed abundance patterns of metal poor stars. Numerical calculations of the multicolor light curves are performed using the multigroup radiation hydrodynamic code STELLA. The calculated light curves of metal-free SNe are compared with our calculations of non-zero metallicity models and observed SNe.
\end{abstract}

Keywords. radiative transfer, shock waves, stars: abundances, supernovae: general, stars: Population III

\section{Overview}

Blue supergiants are typical presupernovae for Pop III core-collapse SNe with $M_{\mathrm{MS}} \lesssim$ 40-60 $\mathrm{M}_{\odot}$ and their structure determines the properties of the light curves. We found that first SNe are bluer, shorter and fainter than ordinary SNe. The most important result is the flat color evolution curve with typical values B-V from 0.0 to 0.6 during the plateau phase can be used as an indicator of Pop III and low-metallicity SNe. In contrast, all solar metallicity models show gradual reddening during the plateau. The low amount of ${ }^{56} \mathrm{Ni}$ that is used to explain carbon-enhanced metal-poor stars with mixing-fallback and sharp luminosity decline after the plateau phase can also be used as indicators of a low-metallicity progenitor.

Our results can be used to identify first-generation SNe in the current (Subaru/HSC) and future transient surveys (LSST, JWST). They are also suitable for identifying of the low-metallicity SNe in the nearby Universe (PTF, Pan-STARRS, Gaia). 